\title{
AN EXTENSION OF THE TOPSIS FOR MULTI-ATTRIBUTE GROUP DECISION MAKING UNDER NEUTROSOPHIC ENVIRONMENT
}

\author{
AMIRHOSSEIN NAFEI, YONGYI GU, AND WENJUN YUAN \\ Received 04 May, 2020
}

\begin{abstract}
Neutrosophic set theory as a generalization of the fuzzy set theory and intuitionistic fuzzy set theory is an effective tool to deal with inconsistent, imprecise, and vague information. TOPSIS is a multiple attribute method to identify solutions from a finite set of alternatives based upon simultaneous minimization of distance from an ideal point and maximization of distance from a nadir point. In this paper, we first develop a new Hamming distance between single-valued neutrosophic numbers and then present an extension of the TOPSIS method for multi-attribute group decision-making (MAGDM) based on single-valued neutrosophic sets, where the inform ation about attribute values and attribute weights are expressed by decision-makers based on neutrosophic numbers.
\end{abstract}

2010 Mathematics Subject Classification: 90B50; 35M10

Keywords: neutrosophic, selection method, decision making, multiple attribute, distance function

\section{SECTION HEAD}

Multi-attribute decision making (MADM) as a component of decision science is a substantial and essential part of daily life which can be applied in various areas, such as society, economics, management, military, and engineering technology. In most cases, it is intricate for decision-makers to accurately reveal a preference when solving MADM problems with imprecise, vague or incomplete information. Under these conditions, in the past few decades, various types of sets, such as fuzzy sets [32], interval-valued fuzzy sets [33], intuitionistic fuzzy sets [1,3], interval-valued intuitionistic fuzzy sets [2], type 2 fuzzy sets [8,11], type $n$ fuzzy sets [8], hesitant fuzzy sets [29] and neutrosophic set theory [26], have been introduced and widely used in the solution of significant decision-making problems. The neutrosophic set theory which is an extension of the intuitionistic fuzzy set provides a practical tool to deal with indeterminate and inconsistent information that exist commonly in the real conditions. A given neutrosophic set such as $N$ has three independent components,

This work is supported by the National Natural Science Foundation (NSF) of China (11901111). 
namely the truth membership $T_{N}(x)$, the indeterminacy membership $I_{N}(x)$ and falsitymembership $F_{N}(x)$.

The technique for order performance by similarity to ideal solution (TOPSIS) was first developed by Hwang and Yoon [11] for solving a MADM problem. It bases upon the concept that the chosen alternative should have the shortest distance from the positive ideal solution (PIS) and the farthest from the negative ideal solution (NIS). In the process of TOPSIS, the performance ratings and the weights of the criteria are given as crisp values. In recent years a lot of MADM methods [4, 14-16,30,31] and multi-attribute group decision making (MAGDM) methods [6, 17, 19] based on the extension of the TOPSIS method have been proposed.

In order to evaluate human resources, Jin et al. [14] introduced an extended TOPSIS method for MADM based on intuitionistic fuzzy sets where the attribute values given by decision-makers are the intuitionistic fuzzy numbers. Wei and Liu [31] presented an extended TOPSIS method based on uncertain linguistic variables to manage high technological risks. In order to resolve MADM problems, Liu in [16] presents an extension of the TOPSIS method where the weights and decision values of the alternatives are considered as interval vague values. Liu and Su [15] proposed an extended TOPSIS based on trapezoid fuzzy linguistic numbers and present a method for determining attribute weights. Rădulescu. C. and Rădulescu. I. [24] by modifying the variable $\rho$ in the Minkowski distance measure proposed an extended TOPSIS method for ranking cloud service providers. Verma et al. [30] proposed an interval-valued intuitionistic fuzzy TOPSIS method for solving a facility location problem. Balin [4] proposed an extension of TOPSIS based on interval-valued spherical fuzzy sets to select the most effective stabilizing system for naval ships. In [6] Chen proposed a symmetric approach to extend the TOPSIS to the fuzzy environment for MAGDM problems in which the weights of various attributes and ratings of alternatives in regard to the different attributes indicated by linguistic variables. By defining a distance formula of generalized interval-valued fuzzy numbers in [17] Liu proposed an extended TOPSIS method for MAGDM problems where the attribute values and weights given by different decision-makers are all generalized intervalvalued fuzzy numbers. In this respect, to choose adequate security mechanisms in ebusiness processes, Mohammadi et al. [19] proposed a fuzzy TOPSIS method based on group recommendation.

In this research, we first develop a distance measure to calculate the distance between single-valued neutrosophic numbers and then present an extended TOPSIS method for MAGDM under the neutrosophic environment where the attribute values and weights given by decision-makers (DMs) are represented by single-valued neutrosophic numbers (SVNNs). The key of our proposed method is that the different neutrosophic decision matrices presented by different decision-makers are converted into a single matrix and create an aggregated group decision matrix. The remaining of this research is marshaled as follows: in the next section, we will briefly review the 
basic concepts of neutrosophic sets, the operation rules of single-valued neutrosophic sets, and the distance between them. Section 3 presents a distance measure to calculate the distance between SVNNs and describes the steps of the proposed method to rank the alternatives. Section 4 gives a numerical example to explain the validity of the proposed method. The study is concluded in Section 5.

\section{PRELIMINARIES}

This section provides a brief review of particular preliminaries regarding neutrosophic sets, the distance between neutrosophic sets (NSs) and some other important concepts.

Definition 1 ([22,27]). A neutrosophic set (NS) $N$ in a domain $X$ (finite universe of objectives) can be represented by $\left.T_{N}: X \rightarrow\right] 0^{-}, 1^{+}\left[, I_{N}: X \rightarrow\right] 0^{-}, 1^{+}[$and $\left.F_{N}: X \rightarrow\right] 0^{-}, 1^{+}$that satisfy the condition $0^{-} \leq T_{N}(x)+I_{N}(x)+F_{N}(x) \leq 3^{+} \forall x \in X$. Where $T_{N}(x), I_{N}(x)$ and $F_{N}(x)$ denote the truth, indeterminacy and falsity membership functions, respectively.

Definition 2 ([20,21]). A neutrosophic set $N$ is contained in another neutrosophic set $M$, if and only if:

$$
\begin{aligned}
\operatorname{Inf} T_{N}(x) & \leq \operatorname{Inf} T_{M}(x), \\
\operatorname{Sup} T_{N}(x) & \leq \operatorname{Sup}_{M}(x), \\
\operatorname{Inf} I_{N}(x) & \geq \operatorname{Inf} I_{M}(x), \\
\operatorname{Sup}_{N}(x) & \geq \operatorname{SupI}_{M}(x), \\
\operatorname{Inf}_{N}(x) & \geq \operatorname{Inf} F_{M}(x), \\
\operatorname{Sup}_{N}(x) & \geq \operatorname{Sup}_{M}(x),
\end{aligned}
$$

for all $x \in X$.

Definition 3 ([25]). The complement of a neutrosophic set $N$ is denoted by $N^{c}$ and can be defined as $T_{N}^{c}(x)=\{1\} \ominus T_{N}(x), I_{N}^{c}(x)=\{1\} \ominus I_{N}(x)$ and $F_{N}^{c}(x)=\{1\} \ominus F_{N}(x)$ for all $x \in X$.

Definition $4([18,28])$. Let $X$ be a domain. A single-valued neutrosophic set (SVNS) $N$ in the domain $X$ can be denoted as $N=\left\{x, T_{N}(x), I_{N}(x), F_{N}(x) ; x \in X\right\}$, where $T_{N}: X \rightarrow[0,1], I_{N}: X \rightarrow[0,1]$ and $F_{N}: X \rightarrow[0,1]$ are three maps in $X$ that satisfy the condition $0 \leq T_{N}(x)+F_{N}(x)+I_{N}(x) \leq 3 \forall x \in X$. The numbers $T_{N}(x), F_{N}(x)$ and $I_{N}(x)$ are the degree of truth, falsity and indeterminacy membership of element $x$ to $N$, respectively.

Remark 1. For a SVNS $N$, the trinary $\left(T_{N}(x), I_{N}(x), F_{N}(x)\right)$ is called a singlevalued neutrosophic number (SVNN). For convenience, the trinary $\left(T_{N}(x), I_{N}(x)\right.$, $\left.F_{N}(x)\right)$ is often denoted by $(T, I, F)$. 
Definition $5([9,18])$. Let $x=\left(T_{1}, I_{1}, F_{1}\right)$ and $y=\left(T_{2}, I_{2}, F_{2}\right)$ be two SVNNs. The mathematical operations between $x$ and $y$ are defined as follows:

$$
\begin{aligned}
\text { I. } & x \oplus y=\left(T_{1}+T_{2}-T_{1} T_{2}, I_{1} I_{2}, F_{1} F_{2}\right), \\
\text { II. } & x \otimes y=\left(T_{1} T_{2}, I_{1}+I_{2}-I_{1} I_{2}, F_{1}+F_{2}-F_{1} F_{2}\right), \\
\text { III. } & \lambda x=\left(1-\left(1-T_{1}\right)^{\lambda}, I_{1}^{\lambda}, F_{1}^{\lambda}\right), \lambda>0, \\
\text { IV. } & x^{\lambda}=\left(T_{1}^{\lambda}, 1-\left(1-I_{1}\right)^{\lambda}, 1-\left(1-F_{1}\right)^{\lambda}\right), \lambda>0 .
\end{aligned}
$$

Definition $6([10,13])$. The complement of a SVNS $N$ is denoted by $N^{c}$ and is defined as $T_{N}^{c}(x)=F_{N}(x), I_{N}^{c}(x)=1-I(x)$ and $F_{N}^{c}(x)=T_{N}(x)$ for all $x \in X$. Therefore $\left[N^{c}=\left\{x, F_{N}(x), 1-I_{N}(x), T_{N}(x) ; x \in X\right\}.\right]$

Definition 7 ([7,12]). Let $N=\left\{x, T_{N}(x), I_{N}(x), F_{N}(x) ; x \in X\right\}$ and $M=\left\{x, T_{M}(x)\right.$, $\left.I_{M}(x), F_{M}(x) ; x \in X\right\}$ be two single-valued neutrosophic sets, the Hamming distance between $N$ and $M$ is defined as follow:

$$
d_{H}(x, y)=\frac{1}{6}\left(\left|T_{N}(x)-T_{M}(x)\right|+\left|I_{N}(x)-I_{M}(x)\right|+\left|F_{N}(x)-F_{M}(x)\right|\right),
$$

also, the Euclidian distance between $N$ and $M$ is defined as follow:

$$
d_{E}(N, M)=\sqrt{\frac{1}{6}\left(\left(T_{N}(x)-T_{M}(x)\right)^{2}+\left(I_{N}(x)-I_{M}(x)\right)^{2}+\left(F_{N}(x)-F_{M}(x)\right)^{2}\right)} .
$$

Definition $8([5,23]), d(N, M)$ is said to be a distance measure between neutrosophic sets if it satisfies the following properties:

P1: $d(N, M) \geq 0$.

P2: $d(N, M)=0$ if and only if $N=M$ for all $N, M \in$ NSs.

P3: $d(N, M)=d(M, N)$.

P4: If $N \subseteq M \subseteq O$ where $O \in$ NSs in $X$ then: $d(N, O) \geq d(N, M)$ and $d(N, O) \geq d(M, O)$.

\section{THE PROPOSED METHOD}

In this section, we first propose a new Hamming distance based on the Hausdorff metric between single-valued neutrosophic numbers. Then we will use this distance to present a new multi-attribute group decision-making method (MAGDM) based on the combination of neutrosophic sets and extended TOPSIS method.

\subsection{Extended Hausdorff distance}

Let $X=\left\{x_{1}, x_{2}, \ldots, x_{n}\right\}$ be a finite universe of objectives. Consider two neutrosophic sets $N$ and $M$ in $X$ where $N=\left\{x_{i}, T_{N}\left(x_{i}\right), I_{N}\left(x_{i}\right), F_{N}\left(x_{i}\right) ; x_{i} \in X\right\}$ and $M=\left\{x_{i}, T_{M}\left(x_{i}\right), I_{M}\left(x_{i}\right), F_{M}\left(x_{i}\right) ; x_{i} \in X\right\}$. Then denote

$$
d(N, M)=\frac{1}{n} \sum_{i=1}^{n}\left[\frac{\left(\left|T_{N}\left(x_{i}\right)-T_{M}\left(x_{i}\right)\right|+\left|I_{N}\left(x_{i}\right)-I_{M}\left(x_{i}\right)\right|+\left|F_{N}\left(x_{i}\right)-F_{M}\left(x_{i}\right)\right|\right)}{6}\right.
$$




$$
\left.+\frac{\max \left(\left|T_{N}\left(x_{i}\right)-T_{M}\left(x_{i}\right)\right|,\left|I_{N}\left(x_{i}\right)-I_{M}\left(x_{i}\right)\right|,\left|F_{N}\left(x_{i}\right)-F_{M}\left(x_{i}\right)\right|\right)}{3}\right] .
$$

Theorem 1. $d(N, M)$ is a distance between two neutrosophic sets $N$ and $M$ in $X$.

Proof. It is obvious $d(N, M)$ satisfies P1-P3 of Definition 8 . Therefore we only need to prove $d(N, M)$ satisfies $\mathrm{P} 4$. To this aim let $O=\left\{x, T_{O}(x), I_{O}(x), F_{O}(x) ; x \in X\right\}$ be another neutrosophic set. In this case, if $N \subseteq M \subseteq O$ then we have:

$$
\begin{aligned}
d(N, M)= & \frac{1}{n} \sum_{i=1}^{n}\left[\frac{\left(\left|T_{N}\left(x_{i}\right)-T_{M}\left(x_{i}\right)\right|+\left|I_{N}\left(x_{i}\right)-I_{M}\left(x_{i}\right)\right|+\left|F_{N}\left(x_{i}\right)-F_{M}\left(x_{i}\right)\right|\right)}{6}\right. \\
& \left.+\frac{\max \left(\left|T_{N}\left(x_{i}\right)-T_{M}\left(x_{i}\right)\right|,\left|I_{N}\left(x_{i}\right)-I_{M}\left(x_{i}\right)\right|,\left|F_{N}\left(x_{i}\right)-F_{M}\left(x_{i}\right)\right|\right)}{3}\right],
\end{aligned}
$$

and

$$
\begin{aligned}
d(N, O)= & \frac{1}{n} \sum_{i=1}^{n}\left[\frac{\left(\left|T_{N}\left(x_{i}\right)-T_{O}\left(x_{i}\right)\right|+\left|I_{N}\left(x_{i}\right)-I_{O}\left(x_{i}\right)\right|+\left|F_{N}\left(x_{i}\right)-F_{O}\left(x_{i}\right)\right|\right)}{6}\right. \\
& \left.+\frac{\max \left(\left|T_{N}\left(x_{i}\right)-T_{O}\left(x_{i}\right)\right|,\left|I_{N}\left(x_{i}\right)-I_{O}\left(x_{i}\right)\right|,\left|F_{N}\left(x_{i}\right)-F_{O}\left(x_{i}\right)\right|\right)}{3}\right] .
\end{aligned}
$$

It's easy to see

$$
\begin{aligned}
\left|T_{N}\left(x_{i}\right)-T_{O}\left(x_{i}\right)\right| & \geq\left|T_{N}\left(x_{i}\right)-T_{M}\left(x_{i}\right)\right|, \\
\left|I_{N}\left(x_{i}\right)-I_{O}\left(x_{i}\right)\right| & \geq\left|I_{N}\left(x_{i}\right)-I_{M}\left(x_{i}\right)\right|, \\
\left|F_{N}\left(x_{i}\right)-F_{O}\left(x_{i}\right)\right| & \geq\left|F_{N}\left(x_{i}\right)-F_{M}\left(x_{i}\right)\right|,
\end{aligned}
$$

so we have:

$$
\begin{aligned}
& \frac{\left(\left|T_{N}\left(x_{i}\right)-T_{O}\left(x_{i}\right)\right|+\left|I_{N}\left(x_{i}\right)-I_{O}\left(x_{i}\right)\right|+\left|F_{N}\left(x_{i}\right)-F_{O}\left(x_{i}\right)\right|\right)}{6} \\
& \quad+\frac{\max \left(\left|T_{N}\left(x_{i}\right)-T_{O}\left(x_{i}\right)\right|,\left|I_{N}\left(x_{i}\right)-I_{O}\left(x_{i}\right)\right|,\left|F_{N}\left(x_{i}\right)-F_{O}\left(x_{i}\right)\right|\right)}{3} \\
& \geq \frac{\left(\left|T_{N}\left(x_{i}\right)-T_{M}\left(x_{i}\right)\right|+\left|I_{N}\left(x_{i}\right)-I_{M}\left(x_{i}\right)\right|+\left|F_{N}\left(x_{i}\right)-F_{M}\left(x_{i}\right)\right|\right)}{6} \\
& \quad+\frac{\max \left(\left|T_{N}\left(x_{i}\right)-T_{M}\left(x_{i}\right)\right|,\left|I_{N}\left(x_{i}\right)-I_{M}\left(x_{i}\right)\right|,\left|F_{N}\left(x_{i}\right)-F_{M}\left(x_{i}\right)\right|\right)}{3} .
\end{aligned}
$$

Therefore we can get the inequality $d(N, O) \geq d(N, M)$. By the same reason we can get $d(N, O) \geq d(M, O)$. So $d(N, M)$ satisfies $\mathrm{P} 4$ of Definition 8. That is to say, $d(N, M)$ is a distance measure between neutrosophic sets $N$ and $M$.

\subsection{The extended TOPSIS method for multi-attribute group decision-making}

Suppose that $A=\left\{A_{1}, A_{2}, \ldots, A_{n}\right\}$ be a set of alternatives, $B=\left\{C_{1}, C_{2}, \ldots, C_{m}\right\}$ be a set of attributes and $D=\left\{D_{1}, D_{2}, \ldots D_{k}\right\}$ be a set of decision-makers (DMs). Let $\bar{w}_{p}=\left[\bar{w}_{1}^{p}, \bar{w}_{2}^{p}, \ldots, \bar{w}_{m}^{p}\right]$ be a vector of weights for attributes determined by DM $D_{p}$ 
where $\bar{w}_{j}^{p}$ is a single-valued neutrosophic number denoting the weight of attribute $C_{j}$ given by decision-maker $D_{p} .1 \leq j \leq m$ and $1 \leq p \leq k$.

Assume that $W_{p}$ represents the weight of DM $D_{p}$. If a decision group has $k$ members then $W_{P}=\frac{1}{k}$, where $W_{p} \in[0,1]$ and $\sum_{p=1}^{k} W_{p}=1$.

Let $X_{p}=\left[x_{i j}\right]_{m \times n}$ be a decision matrix of the $n$ alternatives in regard to the $m$ attributes characterized by decision-maker $D_{p}$, shown as follows:

$$
X_{p}=\begin{gathered}
C_{1} \\
A_{1} \\
A_{2} \\
\vdots \\
A_{n}
\end{gathered}\left[\begin{array}{cccc}
x_{11}^{p} & x_{12}^{p} & \ldots & C_{m} \\
x_{21}^{p} & x_{22}^{p} & \ldots & x_{1 m}^{p} \\
\vdots & \vdots & \ddots & \vdots \\
x_{n 1}^{p} & x_{n 2}^{p} & \ldots & x_{n m}^{p}
\end{array}\right],
$$

where $x_{i j}=\left(T_{i j}, I_{i j}, F_{i j}\right)$ is a single value neutrosophic number for the alternative $A_{i}$ in regard to the attribute $C_{j}$.

The procedure of our proposed method can be summarized as follows:

Step 1. According to the weighting vector $\bar{w}_{p}$, the decision matrix $X_{P}$ and the multiplication operator of SVNSs presented in (2.3) calculate the weighted decision matrix (WDM) $E V_{P}$ as follows:

$$
E V_{p}=\begin{array}{ccccc}
A_{1} & C_{2} & \ldots & C_{m} \\
A_{2} \\
\vdots \\
A_{n}
\end{array}\left[\begin{array}{cccc}
x_{11}^{p} \otimes \bar{w}_{1}^{P} & x_{12}^{p} \otimes \bar{w}_{2}^{P} & \ldots & x_{1 m}^{p} \otimes \bar{w}_{m}^{P} \\
x_{21}^{p} \otimes \bar{w}_{1}^{P} & x_{22}^{p} \otimes \bar{w}_{2}^{P} & \ldots & x_{2 m}^{p} \otimes \bar{w}_{m}^{P} \\
\vdots & \vdots & \ddots & \vdots \\
x_{n 1}^{p} \otimes \bar{w}_{1}^{P} & x_{n 2}^{p} \otimes \bar{w}_{2}^{P} & \ldots & x_{n m}^{p} \otimes \bar{w}_{m}^{P}
\end{array}\right]=\left[\begin{array}{cccc}
C_{1} & C_{2} & \cdots & C_{m} \\
y_{11}^{p} & y_{12}^{p} & \ldots & y_{1 m}^{p} \\
y_{21}^{p} & y_{22}^{p} & \ldots & y_{2 m}^{p} \\
\vdots & \vdots & \ddots & \vdots \\
y_{n 1}^{p} & y_{n 2}^{p} & \ldots & y_{n m}^{p}
\end{array}\right] .
$$

Step 2. Based on the obtained WDMs and the weight of decision-makers we can get the aggregated group decision matrix $A G$ of all decision-makers $D_{1}, D_{2}, \ldots, D_{k}$ as follows:

$$
A G=\begin{gathered}
D_{1} \\
A_{2} \\
\vdots \\
A_{n}
\end{gathered}\left[\begin{array}{cccc}
G_{11} & G_{12} & \cdots & D_{1 k} \\
G_{21} & G_{22} & \cdots & G_{2 k} \\
\vdots & \vdots & \ddots & \vdots \\
G_{n 1} & G_{n 2} & \cdots & G_{n k}
\end{array}\right],
$$

where $G_{i p}$ is a neutrosophic value, representing the sum of alternatives in regard to DM $D_{p}$, and can be calculated as follows:

$$
G_{i p}=W_{P}\left[y_{i 1}^{p} \oplus y_{i 2}^{p} \oplus \cdots \oplus y_{i m}^{p}\right],
$$

where $W_{P}$ is the weight of decision-maker $D_{P}$ and $\oplus$ is the addition operator presented in (2.2).

Step 3. Based on the obtained aggregated group decision matrix we know that the elements $G_{i p}$ are SVNNs. The absolute neutrosophic positive ideal solution (NPIS) 
$P^{+}$and the neutrosophic negative ideal solution (NNIS) $P^{-}$can be defined as follows:

$$
\begin{aligned}
& P^{+}=\left(G_{1}^{+}, G_{2}^{+}, \ldots, G_{k}^{+}\right), \\
& P^{-}=\left(G_{1}^{-}, G_{2}^{-}, \ldots, G_{k}^{-}\right),
\end{aligned}
$$

where $G_{j}^{+}=(1,0,0)$ and $G_{j}^{-}=(0,1,1), j=1,2, ., k$. Also we can select the virtual positive ideal solution and negative ideal solution by selecting the best values for each attribute from all alternatives as follows:

$$
\left\{\begin{array}{l}
G_{j}^{+}=\left(\max _{i} T_{i j}, \min _{i} I_{i j}, \min _{i} F_{i j}\right)=\left(T_{j}^{+}, I_{j}^{+}, F_{j}^{+}\right), \quad 1 \leq j \leq k . \\
\left.G_{j}^{-}=\left(\min _{i} T_{i j}, \max _{i} I_{i j}, \max _{i} F_{i j}\right)=\left(T_{j}^{-}, I_{j}^{-}, F_{j}^{-}\right), \quad 1 \leq 1\right)
\end{array}\right.
$$

Step 4. Based on the proposed distance measure in (3.1). calculate the distance between alternative $A_{i}$ and the elements in the obtained positive ideal solution $P^{+}$as follows:

$$
\begin{aligned}
d_{i}^{+}=\sum_{j=1}^{n} G_{i j}-G_{j}^{+}= & \frac{1}{n} \sum_{j=1}^{n}\left[\frac{\left(\left|T_{i j}-T_{j}^{+}\right|+\left|I_{i j}-I_{j}^{+}\right|+\left|F_{i j}-F_{j}^{+}\right|\right)}{6}\right. \\
& \left.+\frac{\max \left(\left|T_{i j}-T_{j}^{+}\right|,\left|I_{i j}-I_{j}^{+}\right|,\left|F_{i j}-F_{j}^{+}\right|\right)}{3}\right],
\end{aligned}
$$

also, the degree of distance between the alternative $A_{i}$ and the elements in the obtained negative ideal solution $P^{-}$can be calculated as follows:

$$
\begin{aligned}
d_{i}^{-}=\sum_{j=1}^{n} G_{i j}-G_{j}^{-}= & \frac{1}{n} \sum_{j=1}^{n}\left[\frac{\left(\left|T_{i j}-T_{j}^{-}\right|+\left|I_{i j}-I_{j}^{-}\right|+\left|F_{i j}-F_{j}^{-}\right|\right)}{6}\right. \\
& \left.+\frac{\max \left(\left|T_{i j}-T_{j}^{-}\right|,\left|I_{i j}-I_{j}^{-}\right|,\left|F_{i j}-F_{j}^{-}\right|\right)}{3}\right],
\end{aligned}
$$

where $1 \leq \mathrm{i} \leq n, 1 \leq \mathrm{j} \leq k$.

Step 5. Compute the relative closeness coefficient to choose the most appropriate and efficient decision by ranking the alternatives as follows:

$$
R_{i}^{*}=\frac{d_{i}^{-}}{d_{i}^{+}+d_{i}^{-}}, 1, \ldots, n .
$$

Step 6. Utilize the relative closeness coefficients to sort the alternatives. The bigger $R_{i}^{*}$ is, the better alternative $A_{i}$ is.

\section{ILLUSTRATIVE EXAMPLE}

In this section, an example based on TOPSIS method for MAGDM under the neutrosophic environment is used as a demonstration of the applications and the effectiveness of the proposed decision-making method. 
Suppose that there is a panel to compare four car companies $A_{1}, A_{2}, A_{3}$ and $A_{4}$ as the alternatives. Also assume that three attributes such as "Quality $\left(C_{1}\right)$ ", "Design $\left(C_{2}\right)$ " and "Price $\left(C_{3}\right)$ ". A committee of three decision-makers $D_{1}, D_{2}$ and $D_{3}$ has been formed to rank the alternatives and choose the best company. Assume that the decision values of company alternatives $A_{1}, A_{2}, A_{3}$ and $A_{4}$ in regard to the attributes "Quality", "Design" and "Price" given by the decision-makers $D_{1}, D_{2}$ and $D_{3}$ based on single-valued neutrosophic numbers, as shown in Table 1, Table 2 and Table 3, respectively.

TABLE 1 . The decision values given by $D_{1}$

\begin{tabular}{llll}
\hline & Quality & Design & Price \\
\hline$A_{1}$ & $(0.1771,0.5573,0.5013)$ & $(0.1079,0.3390,0.4857)$ & $(0.1932,0.6289,0.9274)$ \\
$A_{2}$ & $(0.8296,0.7725,0.4317)$ & $(0.1822,0.2101,0.8944)$ & $(0.8959,0.1015,0.9175)$ \\
$A_{3}$ & $(0.7669,0.3119,0.9976)$ & $(0.0991,0.5102,0.1375)$ & $(0.0991,0.3909,0.7136)$ \\
$A_{4}$ & $(0.9345,0.1790,0.8116)$ & $(0.4898,0.9064,0.3900)$ & $(0.0442,0.0546,0.6183)$ \\
\hline
\end{tabular}

TABLE 2. The decision values given by $D_{2}$

\begin{tabular}{llll}
\hline & Quality & Design & Price \\
\hline$A_{1}$ & $(0.3433,0.5493,0.9542)$ & $(0.6465,0.7565,0.2815)$ & $(0.8352,0.9727,0.5906)$ \\
$A_{2}$ & $(0.9360,0.3304,0.0319)$ & $(0.8332,0.4139,0.2304)$ & $(0.3225,0.3278,0.6604)$ \\
$A_{3}$ & $(0.1248,0.6195,0.3369)$ & $(0.3983,0.4923,0.7111)$ & $(0.5523,0.8378,0.0476)$ \\
$A_{4}$ & $(0.7306,0.3606,0.6627)$ & $(0.7498,0.6947,0.6246)$ & $(0.9791,0.7391,0.3488)$ \\
\hline
\end{tabular}

TABLE 3. The decision values given by $D_{3}$

\begin{tabular}{llll}
\hline & Quality & Design & Price \\
\hline$A_{1}$ & $(0.4513,0.5038,0.3610)$ & $(0.2815,0.4494,0.0839)$ & $(0.1386,0.1892,0.4035)$ \\
$A_{2}$ & $(0.2409,0.4896,0.6203)$ & $(0.7311,0.9635,0.9748)$ & $(0.5882,0.6671,0.1220)$ \\
$A_{3}$ & $(0.2409,0.8770,0.8112)$ & $(0.1378,0.0423,0.6513)$ & $(0.3662,0.5864,0.2684)$ \\
$A_{4}$ & $(0.8562,0.3531,0.0193)$ & $(0.8367,0.9730,0.2312)$ & $(0.8068,0.6751,0.2578)$ \\
\hline
\end{tabular}

Therefore the corresponding decision matrices $X_{1}, X_{2}$ and $X_{3}$ can be shown as follows, respectively:

$$
X_{1}=\left[\begin{array}{cc}
C_{1} & C_{2} \\
(0.1771,0.5573,0.5013),(0.1079,0.3390,0.4857),(0.1932,0.6289,0.9274) \\
(0.8296,0.7725,0.4317),(0.1822,0.2101,0.8944),(0.8959,0.1015,0.9175) \\
(0.7669,0.3119,0.9976),(0.0991,0.5102,0.1375),(0.0991,0.3909,0.7136) \\
(0.9345,0.1790,0.8116),(0.4898,0.9064,0.3900),(0.0442,0.0546,0.6183)
\end{array}\right],
$$




$$
\begin{aligned}
& C_{1} \quad C_{2} \quad C_{3} \\
& X_{2}=\left[\begin{array}{c}
(0.3433,0.5493,0.9542),(0.6465,0.7565,0.2815),(0.8352,0.9727,0.5906) \\
(0.9360,0.3304,0.0319),(0.8332,0.4139,0.2304),(0.3225,0.3278,0.6604) \\
(0.1248,0.6195,0.3369),(0.3983,0.4923,0.7111),(0.5523,0.8378,0.0476) \\
(0.7306,0.3606,0.6627),(0.7498,0.6947,0.6246),(0.9791,0.7391,0.3488)
\end{array}\right], \\
& C_{1} \quad C_{2} \quad C_{3} \\
& X_{3}=\left[\begin{array}{l}
(0.4513,0.5038,0.3610),(0.2815,0.4494,0.0839),(0.1386,0.1892,0.4035) \\
(0.2409,0.4896,0.6203),(0.7311,0.9635,0.9748),(0.5882,0.6671,0.1220) \\
(0.2409,0.8770,0.8112),(0.1378,0.0423,0.6513),(0.3662,0.5864,0.2684) \\
(0.8562,0.3531,0.0193),(0.8367,0.9730,0.2312),(0.8068,0.6751,0.2578)
\end{array}\right] .
\end{aligned}
$$

Suppose that the attribute weights given by three DMs $D_{1}, D_{2}$ and $D_{3}$ are shown as follows:

$$
C_{1} \quad C_{2} \quad C_{3}
$$

$W_{1}=[(0.2834,0.3900,0.8344),(0.8962,0.4979,0.6096),(0.8266,0.6948,0.5747)]$,

$$
C_{1} \quad C_{2} \quad C_{3}
$$

$W_{2}=[(0.3260,0.8844,0.6748),(0.4564,0.7209,0.4385),(0.7138,0.0186,0.4378)]$,

$C_{1} \quad C_{2} \quad C_{3}$

$W_{3}=[(0.1170,0.2462,0.5466),(0.8147,0.3427,0.5619),(0.3249,0.3757,0.3958)]$.

Furthermore, because the decision group in this example has three members we can consider $W_{1}=W_{2}=W_{3}=\frac{1}{3}$.

The proposed method is currently applied to solve this problem and the computational procedure is summarized as follows:

Step 1. Construct the weighted decision matrices $E V_{1}, E V_{2}$ and $E V_{3}$ as follows:

$$
\begin{gathered}
E V_{1}=\left[\begin{array}{c}
C_{1} \\
(0.0502,0.7300,0.9174),(0.0967,0.6681,0.7992),(0.1597,0.8867,0.9691) \\
(0.2351,0.8612,0.9059),(0.1633,0.6034,0.9588),(0.7405,0.7258,0.9649) \\
(0.2173,0.5803,0.9996),(0.0888,0.7540,0.6633),(0.0819,0.8141,0.8782) \\
(0.2648,0.4992,0.9688),(0.4389,0.9530,0.7619),(0.0365,0.7115,0.8377)
\end{array}\right], \\
C_{1} \\
C_{2} \\
E V_{2}=\left[\begin{array}{c}
(0.1119,0.9479,0.9851),(0.2951,0.9320,0.5966),(0.5962,0.9732,0.7698) \\
(0.3052,0.9226,0.6852),(0.3803,0.8364,0.5679),(0.2302,0.3403,0.8091) \\
(0.0407,0.9650,0.7908),(0.1818,0.8583,0.8378),(0.3942,0.8408,0.4646) \\
(0.2382,0.9261,0.8903),(0.3422,0.9148,0.7892),(0.6989,0.7439,0.6339)
\end{array}\right],
\end{gathered}
$$




$$
E V_{3}=\left[\begin{array}{cc}
C_{1} & C_{2} \\
(0.0528,0.6260,0.7103),(0.2293,0.6381,0.5987),(0.0450,0.4938,0.6396) \\
(0.0282,0.6153,0.8278),(0.5956,0.9760,0.9890),(0.1911,0.7922,0.4695) \\
(0.0837,0.9073,0.9144),(0.1122,0.3705,0.8473),(0.1189,0.7418,0.5580) \\
(0.1002,0.5124,0.5553),(0.6817,0.9822,0.6632),(0.2621,0.7972,0.5516)
\end{array}\right]
$$

Step 2. Based on the obtained WDMs $E V_{1}, E V_{2}, E V_{3}$ and addition operator of SVNN shown in (2.2) construct the aggregated group decision matrix of all decision-makers as follows:

$$
A G=\left[\begin{array}{cc}
D_{1} & D_{2} \\
(0.0930,0.1442,0.2368),(0.2491,0.2866,0.1508),(0.1010,0.0658,0.0907) \\
(0.2780,0.1257,0.2794),(0.2228,0.0875,0.1049),(0.2274,0.1586,0.1281) \\
(0.1151,0.1187,0.1941),(0.1748,0.2300,0.1026),(0.0944,0.0831,0.1441) \\
(0.2009,0.1128,0.2061),(0.2830,0.2101,0.1485),(0.2629,0.1337,0.0677)
\end{array}\right] .
$$

Step 3. Determine the virtual NPIS and NNIS as:

$$
\begin{aligned}
G_{j}^{+} & =[(0.2780,0.1128,0.1941),(0.2830,0.0875,0.1026),(0.2629,0.0658,0.0677)], \\
G_{j}^{-} & =[(0.0930,0.1442,0.2794),(0.1748,0.2866,0.1508),(0.0944,0.1586,0.1441)] .
\end{aligned}
$$

Step 4. Calculate the distance of each alternative from NPIS and NNIS, respectively, as follows:

$$
\begin{aligned}
& d_{1}^{+}=0.3028, d_{2}^{+}=0.1377, d_{3}^{+}=0.2715, d_{4}^{+}=0.1435 \\
& d_{1}^{-}=0.1148, d_{2}^{-}=0.2799, d_{3}^{-}=0.1246, d_{4}^{-}=0.2397
\end{aligned}
$$

Step5. Based on (3.9), the relative closeness coefficient of each candidate can be calculated as follows:

$$
R_{1}^{*}=0.2748, R_{2}^{*}=0.6703, R_{3}^{*}=0.3146, R_{4}^{*}=0.6255 .
$$

Therefore, the ranking order of the four alternatives is $A_{2}, A_{4}, A_{3}$ and $A_{1}$. Obviously, the best selection is $A_{2}$.

Remark 2. In recent years, a lot of extended TOPSIS methods have been presented to deal with MAGDM problems that only consider crisp or incomplete information on their calculation. But until now there hasn't been any TOPSIS method to consider and handle indeterminate and inconsistent information that exists commonly in real decision-making problems. In order to overcome this drawback, this paper for the first time presents an extended TOPSIS method for MAGDM problems based on a single-valued neutrosophic set. Although by using the neutrosophic sets we are faced with a large class of problems the proposed method has less calculation and is more flexible for decision making in the real world. 


\section{CONCLUSiON}

In general, decision-making problems are included uncertain and imprecise information, and neutrosophic sets can depict this kind of information easier and better. Because TOPSIS is an important decision-making method, and the neutrosophic sets can handle the incomplete, indeterminate and inconsistent data, it is important to establish an extended TOPSIS method based on NSs. In this paper, we first develop a distance measures which is an effective and simple tool to measure the distance between two single-valued neutrosophic numbers and then present an extended TOPSIS method to deal with multi-attribute group decision-making (MAGDM) under neutrosophic environment, where decision-makers express the attribute weights and attribute values for alternatives by using neutrosophic numbers. Although the proposed method presented in this paper is illustrated by a personal selection problem, however, it can also be applied to problems such as information project selection, material selection and many other areas of management decision problems.

\section{REFERENCES}

[1] K. T. Atanassov, "Intuitionistic fuzzy sets," Fuzzy sets and systems, vol. 1, no. 20, pp. 87-96, 1986, doi: 10.1016/S0165-0114(86)80034-3.

[2] K. T. Atanassov, "More on intuitionistic fuzzy sets," Fuzzy sets and systems, vol. 33, no. 1, pp. 37-45, 1989, doi: 10.1016/0165-0114(89)90215-7.

[3] K. T. Atanassov, "Intuitionistic fuzzy sets," in Intuitionistic fuzzy sets. Springer, 1999, pp. 1-137, doi: 10.1007/978-3-7908-1870-3-1.

[4] A. Balin, "A novel fuzzy multi-criteria decision-making methodology based upon the spherical fuzzy sets with a real case study," Iranian Journal of Fuzzy Systems, 2020, doi: 10.22111/ijfs.2020.5234.

[5] S. Benbernou, S. Gala, and M. A. Ragusa, "On the regularity criteria for the 3d magnetohydrodynamic equations via two components in terms of bmo space," Mathematical Methods in the Applied Sciences, vol. 37, no. 15, pp. 2320-2325, 2014, doi: 10.1002/mma.2981.

[6] C.-T. Chen, "Extensions of the TOPSIS for group decision-making under fuzzy environment," Fuzzy sets and systems, vol. 114, no. 1, pp. 1-9, 2000, doi: 10.1016/S0165-0114(97)00377-1.

[7] P. Chi and P. Liu, "An extended TOPSIS method for the multiple attribute decision making problems based on interval neutrosophic set," Neutrosophic Sets and Systems, vol. 1, no. 1, pp. 63-70, 2013, doi: 10.5281/zenodo.571231.

[8] D. Dubois and H. Prade, Fuzzy sets and systems: theory and applications. Academic press, 1980, vol. 144, doi: 10.1057/jors.1982.38.

[9] S. Gala, Q. Liu, and M. A. Ragusa, "A new regularity criterion for the nematic liquid crystal flows," Applicable Analysis, vol. 91, no. 9, pp. 1741-1747, 2012, doi: 10.1080/00036811.2011.581233.

[10] S. Gala and M. A. Ragusa, "Logarithmically improved regularity criterion for the boussinesq equations in Besov spaces with negative indices," Applicable Analysis, vol. 95, no. 6, pp. 12711279, 2016, doi: 10.1080/00036811.2015.1061122.

[11] C. Hwang and K. Yoon, Multiple Attribute Decision Making. Springer, New York, 1981, vol. 186, doi: 10.1007/978-3-642-48318-9.

[12] A. Javadpour, "Improving resources management in network virtualization by utilizing a softwarebased network," Wireless Personal Communications, vol. 106, no. 2, pp. 505-519, 2019, doi: 10.1007/s11277-019-06176-6. 
[13] A. Javadpour, "An optimize-aware target tracking method combining mac layer and active nodes in wireless sensor networks," Wireless Personal Communications, vol. 108, no. 2, pp. 711-728, 2019, doi: 10.1007/s11277-019-06425-8.

[14] F. Jin, L. PD, and X. Zhang, "Evaluation study of human resources based on intuitionistic fuzzy set and TOPSIS method," Journal of Information and Computational Science, vol. 4, no. 3, pp. 1023-1028, 2007.

[15] P. Liu and Y. Su, "The extended TOPSIS based on trapezoid fuzzy linguistic variables," Journal of Convergence Information Technology, vol. 5, no. 4, pp. 38-53, 2010, doi: 10.4156/jcit.vol5.issue4.5.

[16] P. Liu, "Multi-attribute decision-making method research based on interval vague set and TOPSIS method," Technological and economic development of economy, vol. 15, no. 3, pp. 453-463, 2009, doi: 10.3846/1392-8619.2009.15.453-463.

[17] P. Liu, "An extended TOPSIS method for multiple attribute group decision making based on generalized interval-valued trapezoidal fuzzy numbers," Informatica, vol. 35, no. 2, 2011.

[18] P. Liu and Y. Wang, "Multiple attribute decision-making method based on single-valued neutrosophic normalized weighted Bonferroni mean," Neural Computing and Applications, vol. 25, no. 7-8, pp. 2001-2010, 2014, doi: 10.1007/s00521-014-1688-8.

[19] S. Mohammadi, S. Golara, and N. Mousavi, "Selecting adequate security mechanisms in ebusiness processes using fuzzy TOPSIS," International Journal of Fuzzy System Applications (IJFSA), vol. 2, no. 1, pp. 35-53, 2012, doi: 10.4018/ijfsa.2012010103.

[20] A. H. Nafei, S. M. E. P. Azizi, and R. A. Ghasempour, "An approach in solving data envelopment analysis with stochastic data," pp. 154-162, 2016, doi: 10.1007/978-3-319-66514-6-17.

[21] A. H. Nafei, W. Yuan, and H. Nasseri, "Group multi-attribute decision making based on interval neutrosophic sets," Studies in Informatics and Control, vol. 28, no. 3, pp. 309-316, 2019, doi: 10.24846/v28i3y201907.

[22] A. Nafei, Y. Wenjun, and H. NASSERI, "A new method for solving interval neutrosophic linear programming problems," Gazi University Journal of Science, vol. 33, no. 4, pp. 796-808, 2020, doi: 10.35378/gujs.689125.

[23] M. Olfati, W. Yuan, and S. Nasseri, "An integrated model of fuzzy multi-criteria decision making and stochastic programming for the evaluating and ranking of advanced manufacturing technologies," Iranian Journal of Fuzzy Systems, vol. 17, no. 5, pp. 183-196, 2020, doi: 10.22111/ijfs.2020.5524.

[24] C. Z. Rădulescu and I. C. Rădulescu, "An extended TOPSIS approach for ranking cloud service providers," Studies in Informatics and Control, vol. 26, no. 2, pp. 183-192, 2017, doi: 10.24846/v26i2y201706.

[25] R. Şahin, "Multi-criteria neutrosophic decision making method based on score and accuracy functions under neutrosophic environment," arXiv preprint arXiv:1412.5202, 2014, doi: 10.5281/zenodo.22994.

[26] F. Smarandache, "A unifying field in logics. neutrosophy: Neutrosophic probability, set and logic," 1999.

[27] F. Smarandache, A Unifying Field in Logics: Neutrosophic Logic. Neutrosophy, Neutrosophic Set, Neutrosophic Probability: Neutrsophic Logic. Neutrosophy, Neutrosophic Set, Neutrosophic Probability. American Research Press, Rehoboth, 2005.

[28] F. Smarandache, Multispace \& Multistructure. Neutrosophic Transdisciplinarity (100 Collected Papers of Science). North-European Scientific Publishers, 2010, vol. 4.

[29] V. Torra and Y. Narukawa, “On hesitant fuzzy sets and decision,” pp. 1378-1382, 2009, doi: 10.1109/FUZZY.2009.5276884. 
[30] A. Verma, R. Verma, and N. Mahanti, "Facility location selection: an interval valued intuitionistic fuzzy TOPSIS approach," Journal of Modern Mathematics and Statistics, vol. 4, no. 2, pp. 68-72, 2010, doi: 10.3923/jmmstat.2010.68.72.

[31] Y. Wei and P. Liu, "Risk evaluation method of high-technology based on uncertain linguistic variable and TOPSIS method.” JCP, vol. 4, no. 3, pp. 276-282, 2009, doi: 10.4304/jcp.4.3.276282.

[32] L. A. Zadeh, "Fuzzy sets," Information and control, vol. 3, no. 8, pp. 338-353, 1965, doi: 10.1016/S0019-9958(65)90241-X.

[33] L. A. Zadeh, "The concept of a linguistic variable and its application to approximate reasoningIII," Information sciences, vol. 9, no. 1, pp. 43-80, 1975, doi: 10.1016/0020-0255(75)90017-1.

Authors' addresses

AmirHossein Nafei

Department of Mathematics and Information Science, Guangzhou University, Guangzhou, China, 510006

E-mail address: Amir.nafeide.gzhu.edu.cn

Yongyi Gu

Department of Statistics and Mathematics, Guangdong University of Finance and Economics, Guangzhou, China, 510320

E-mail address: gdguyongyi@163.com

Wenjun Yuan

Department of Mathematics and Information Science, Guangzhou University, Guangzhou, China, 510006

E-mail address: wjyuan1957@126.com 